\title{
THE SOLUTION OF EIGENVALUE PROBLEMS OF INTEGRAL EQUATIONS BY POWER SERIES*
}

By J. R. M. RADOK (Dept. of Supply, Aeronautical Research Laboratories, Melbourne, Australia)

Summary. The characteristic equations and eigensolutions of Fredholm integral equations of the second kind with symmetrical kernels of the homogeneous polynomial type are obtained by use of power series. The method is applied to the particular case of the kernel $|x-y|$ and it is indicated that it may readily be extended to more general types of equations.

1. Introduction. One of the standard methods of solution of ordinary linear differential equations makes use of power series. Although certain types of integral equations correspond to such differential equations, to the author's knowledge no equivalent method for the direct solution of these equations has been developed up to date. It is the object of this report to fill this gap.

In [1] a method of solution of Fredholm integral equations of the second kind with symmetrical kernels of the polynomial type has been developed by Heller and Radok. This method was conceived as an iteration process. The only difference from the normal iteration procedure arose from the fact that the initial function, which in the commonly used process is arbitrary, was determined by the kernel of the integral equation. The choice of the initial function was thus based on the observation that the integral operator in the equation transformed an arbitrary power of the integration variable into certain "dominant" terms with exponents independent of the exponent of the power originally substituted and into one term. of higher order. In other words, if the integral operator is given by

$$
L \varphi=\int_{0}^{1} K(x, y) \varphi(y) d y
$$

then

$$
L\left(y^{m}\right)=x^{m+k} c_{m}+\sum_{l=\mu}^{\nu} A_{m l} x^{l},
$$

where $\mu, \nu, k$ only depend on the kernel. Substituting for the initial function $\varphi_{0}$ a polynomial with arbitrary coefficients of the type represented by the "dominant" term in (1.2), applying to this initial function an arbitrary number of iterations and comparing then coefficients of powers of $x$ on both sides of the integral equation

$$
\varphi=\lambda L(\varphi)
$$

the corresponding number of exact terms of the power expansions of the eigensolutions and of the characteristic equation may be obtained.

In this report the above process, which still contains formally some of the disadvantages of iteration processes, will be generalized into a straightforward power series method. In order to simplify the analysis, consideration will here be restricted to kernels of the homogeneous polynomial type. However it should be stressed that the method is easily extended to much wider classes of kernels. In fact, it will become obvious that

*IReceived Feb. 17, 1954. 
it is applicable to kernels involving a finite number of linearly independent functions, in which case the desired eigensolutions have to be expanded in infinite series of the complete system of functions occurring in the kernel. In particular, this will be the case for kernels involving trigonometric functions, when the eigensolutions will be obtained in the form of Fourier series.

The general method of solution will be developed in Sec. 2, while one of the simplest kernels of the special type under consideration will be investigated in Sec. 3, in order to demonstrate the ease with which the method may be applied to actual problems. In Ref. [1], two more complicated types of kernels have been studied for which the exact solutions are known and which arise from the problems of natural vibrations of a uniform beam and of a wedge, clamped at one end.

2. The general method. As indicated in the introduction, consideration will be restricted to Fredholm integral equations of the second kind with symmetrical kernels of the homogeneous polynomial type, i.e., to equations of the type:

$$
\varphi(x)=\lambda L(\varphi)=\lambda\left[\int_{0}^{x} P_{n-1}(x, y) \varphi(y) d y+\int_{x}^{1} P_{n-1}(y, x) \varphi(y) d y\right]
$$

where

$$
P_{n-1}(x, y)=\sum_{(i, k)}^{n-1} A_{i k} x^{i} y^{k}, \quad i+k=n-1 .
$$

If it is assumed that $\varphi(x)$ is continuous and differentiable, it is easily seen that $\varphi$ satisfies a differential equation of the form

$$
\varphi^{(n)}=\lambda \sum_{i=0}^{n-1} p_{i} \varphi^{(i)},
$$

where $p_{i}$ are polynomials in $x$; hence the solutions $\varphi$ must be integral functions, i.e.,

$$
\varphi(x)=\sum_{l=0}^{\infty} a_{l} x^{l},
$$

and the use of power series is justified.

Consider the operator

$$
L(\varphi)=\int_{0}^{x} P_{n-1}(x, y) \varphi(y) d y+\int_{x}^{1} P_{n-1}(y, x) \varphi(y) d y .
$$

In particular,

$$
L\left(y^{l}\right)=c_{l} x^{n+l}+\sum_{k=0}^{n-1} x^{k} A_{k l}^{*}
$$

where

$$
\begin{gathered}
c_{l}=\sum_{(i, k)=0}^{n-1} \frac{(i-k) A_{i k}}{(l+i+1)(l+k+1)}=O\left(\left(\frac{1}{l+1}\right)^{2}\right), \quad l=0,1, \cdots \\
A_{k l}^{*}=\sum_{i=0}^{n-1} \frac{A_{i k}}{l+i+1}=O\left(\frac{1}{l+1}\right), \quad l=0,1, \cdots \\
k=0,1, \cdots, n-1 .
\end{gathered}
$$


Applying the operator (2.5) to (2.3), substituting in (2.1) and comparing coefficients of the powers of $x$, one finds

$$
a_{n+l}=\lambda c_{l} a_{l}, \quad l \geq n
$$

and hence, for $0 \leq k \leq n-1$,

$$
a_{k+\nu n}=\lambda^{\nu} a_{k} \prod_{\mu=0}^{n-1} c_{k+n \mu}, \quad \nu \geq 1 .
$$

Finally, the powers of $x$ for $0 \leq l \leq n-1$ give

$$
\begin{aligned}
a_{l} & =\lambda \sum_{\mu=0}^{n-1} \sum_{\nu=0}^{\infty} A_{l, \mu+\nu n}^{*} a_{\mu+\nu n} \\
& =\lambda \sum_{\mu=0}^{n-1} \sum_{\nu=0}^{\infty} A_{l, \mu+\nu n}^{*} \lambda^{\nu} a_{\mu} \prod_{\kappa=0}^{n-1} c_{\mu+n \kappa}, \quad l=0,1, \cdots, n-1 .
\end{aligned}
$$

In order that the system of equations (2.10) will have non-trivial solutions $a_{\iota}$, one has

$$
\left|\delta_{l_{\mu}}-\lambda G_{l_{\mu}}(\lambda)\right|=0, \quad l, \mu=0,1, \cdots, n-1,
$$

where

$$
G_{l, \mu}(\lambda)=\sum_{\nu=0}^{\infty} A_{l, \mu+\nu n} \lambda^{\nu} \prod_{\kappa=0}^{\nu-1} c_{\mu+n \kappa} .
$$

The determinantal equation (2.11) determines the eigenvalues of the integral equation (2.1), while the corresponding eigensolutions are given by

$$
\varphi_{\lambda}(x)=\sum_{\mu=0}^{n-1} \sum_{\nu=0}^{\infty} \lambda^{\nu} x^{\mu+n \nu} a_{\mu} \prod_{\kappa=0}^{\nu-1} c_{\mu+n \kappa} .
$$

It is easily verified from (2.6) that (2.13) converges with $\nu$ for all $x$, the rate of convergence depending on $\mu$ and $\lambda$. The same is true for the function $G_{l, \mu}(\lambda)$ of (2.12), i.e., for (2.10).

3. Example. As an example of the application of the method of solution of Sec. 2 the following integral equation will be investigated. It is one of the simplest of the type under consideration and in many text books, e.g. Bückner [2], is chosen for the demonstration of methods of solution:

$$
\varphi(x)=\lambda\left[\int_{0}^{x}(x-y) \varphi(y) d y+\int_{x}^{1}(y-x) \varphi(y) d y\right] ;
$$

hence, in the notation of Eq. (2.2),

$$
\begin{gathered}
P_{n-1}(x, y)=x-y, \quad i+k=1, \quad n=2, \\
A_{00}=A_{11}=0, \quad A_{01}=-1, \quad A_{10}=1 .
\end{gathered}
$$

The integral operator in the present case is

$$
L(\varphi)=\int_{0}^{x}(x-y) \varphi(y) d y+\int_{x}^{1}(y-x) \varphi(y) d y
$$


and, in correspondence with (2.5), one has

$$
L\left(y^{l}\right)=\frac{2 x^{l+2}}{(l+1)(l+2)}-\frac{x}{l+1}+\frac{1}{l+2},
$$

since by (2.6)

$$
\begin{gathered}
A_{n^{\prime}}^{*}=\frac{1}{l+2}, \quad A_{1 l}^{*}=-\frac{1}{l+1}, \\
c_{l}=\frac{2}{(l+1)(l+2)} .
\end{gathered}
$$

Proceeding as indicated in Sec. 2, comparison of coefficients of powers of $x$ gives

$$
\begin{gathered}
a_{l+2}=\frac{2 \lambda a_{l}}{(l+1)(l+2)^{\prime}}, \quad l \geq 2, \\
a_{0+2 \nu}=\frac{(2 \lambda)^{\nu} a_{0}}{(2 \nu) !}, \quad a_{1+2 \nu}=\frac{(2 \lambda)^{\nu} a_{1}}{(2 \nu+1) !}, \quad \nu \geq 1, \\
a_{0}=\lambda\left[a_{0} \sum_{\nu=0}^{\infty} \frac{(2 \lambda)^{\nu}}{(2 \nu+2)(2 \nu) !}+a_{1} \sum_{\nu=0}^{\infty} \frac{(2 \lambda)^{\nu}}{(2 \nu+3)(2 \nu+1) !}\right] \\
a_{1}=-\lambda\left[a_{0} \sum_{\nu=0}^{\infty} \frac{(2 \lambda)^{\nu}}{(2 \nu+1) !}+a_{1} \sum_{\nu=0}^{\infty} \frac{(2 \lambda)^{\nu}}{(2 \nu+2) !}\right] .
\end{gathered}
$$

The system of Eqs. (3.8a) which corresponds to (2.10) is easily seen to lead to the determinantal equation

$$
\left|\begin{array}{cc}
1+\cosh \omega-\omega \sinh \omega & \frac{\sinh \omega}{\omega}-\cosh \omega \\
\omega \sinh \omega & 1+\cosh \omega
\end{array}\right|=0
$$

or, finally, to

$$
2+2 \cosh \omega-\omega \sinh \omega=0
$$

where

$$
2 \lambda=\omega^{2} .
$$

The eigensolutions are given by

$$
\begin{aligned}
\varphi & =a_{0} \sum_{\nu=0}^{\infty} \frac{(2 \lambda)^{\nu} x^{2 \nu}}{(2 \nu) !}+a_{1} \sum_{\nu=0}^{\infty} \frac{(2 \lambda)^{\nu} x^{1+2 \nu}}{(2 \nu+1) !} \\
& =a_{0} \cosh \omega x+\frac{a_{1}}{\omega} \sinh \omega x .
\end{aligned}
$$

4. Conclusions. A method has been developed by which the eigenvalues and eigenfunctions of certain types of Fredholm integral equations of the second kind may be 
determined. The method which essentially corresponds to the method of power series, used for ordinary differential equations, may be applied to more general kernels than those of the homogeneous polynomial type considered here. It may also be extended to other systems of linearly independent functions, such as trigonometric functions, depending on the construction of the kernel which in that case would have to involve a finite number of trigonometric terms.

The method should prove of great value in the treatment of vibration problems of systems with polynomial mass distributions and concentrated masses. Two of the simplest problems of this type, whose exact solutions have been known for a large number of years, have been solved by a closely related method in [1].

5. Acknowledgment. The author is indebted to Prof. A. Pfluger at the Eidgenössische Technische Hochschule, Zürich, Switzerland, for some valuable suggestions which greatly assisted the further development of the method.

\section{REFERENCES}

1. A. Heller, J. R. M. Radok, Die exakte Lösung der Integralgleichungen gewisser Schwingungs probleme, Zeitschrift für angewandte Mathematik und Physik, 1954.

2. H. Bückner, Die praktische Behandlung von Integralgleichungen, Springer Verlag, Berlin, 1952.

\section{ASYMMETRICAL FINITE DIFFERENCE NETWORK FOR TENSOR CONDUCTIVITIES*}

\section{By L. TASNY-TSCHIASSNY (U'niversity of Sydney, Australia)}

In a paper recently published in this journal [1] the problem dealt with was described by its author in the following way: "Given a region in which Eq. (4) holds and a large number of points in the region chosen at random, in what way should the points be interconnected with 'physically realizable' electrical resistors in order that the voltages at the nodes shall be as nearly as possible the correct solutions of the boundary value problem characterized by Eq. (4) and appropriate boundary conditions?"

The Eq. (4) quoted is the special case of the differential equation

$$
\nabla \cdot\{\sigma[\epsilon] \cdot \nabla \Phi\}+\tau=0
$$

for $[\epsilon]$ being the unit tensor, i.e., for isotropically conducting material. In Eq. (1) $\Phi$ is the electrical potential in a two-dimensional continuum, $\tau$ the current density of a distributed transverse external source, $\sigma$ the conductivity of the continuum in a certain direction, and $[\epsilon]$ the non-uniformity tensor of the conductivity. The scalars $\tau, \sigma$, and the tensor [ $\epsilon]$ may be functions of the position.

The author of the present Note had been interested in a practically identical problem for which he had coined the expression "triangulation of a two-dimensional continuum" and which included the general case of tensor conductivities [2]. He replaced the interior

*Received March 3, 1954. 